\title{
Down-regulation of SOCS6: An Unfavorable Prognostic Factor for Gastrointestinal Stromal Tumor Proven by Survival Analysis
}

\section{Jun Ouyang}

The Seventh Affiliated Hospital Sun Yat-sen University

Tailai An ( $\boldsymbol{D}$ antailai@mail2.sysu.edu.cn )

The Seventh Affiliated Hospital Sun Yat-sen University https://orcid.org/0000-0003-0666-752X

\section{Yan Wang}

Shenzhen People's Hospital

\section{Xiaofang Lu}

The Seventh Affiliated Hospital Sun Yat-sen University

\section{Yawei Zhang}

The Seventh Affiliated Hospital Sun Yat-sen University

\section{Xiaokun Wang}

The Seventh Affiliated Hospital Sun Yat-sen University

\section{Xinhua Zhang}

Sun Yat-sen University First Affiliated Hospital

\section{Changhua Zhang}

The Seventh Affiliated Hospital Sun Yat-sen University

\section{Research}

Keywords: Gastrointestinal stromal tumor, SOCS6, Overall survival, Recurrence-free survival, Cox proportional regression model analysis

Posted Date: April 13th, 2021

DOl: https://doi.org/10.21203/rs.3.rs-414295/v1

License: (c) (i) This work is licensed under a Creative Commons Attribution 4.0 International License. Read Full License

Version of Record: A version of this preprint was published at Diagnostic Pathology on December 1st, 2021. See the published version at https://doi.org/10.1186/s13000-021-01172-6. 


\section{Abstract}

Background: Multiple studies reporting that down-regulation of SOCS6 plays vital roles in promoting progression of many tumors have been published. The present study was performed to identify whether SOCS6 was an biomarker significantly associated with prognosis of patients diagnosed with gastrointestinal stromal tumor (GIST).

Methods: Immunohistochemical staining was accomplished to evaluate the expression levels of SOCS6 among GIST patients. The impacts of SOCS6 expression on overall survival (OS) and recurrence-free survival (RFS) of GIST patients were assessed by Cox proportional hazard regression model analysis and Kaplan-Meier curve analysis.

Results: It was demonstrated that the expression level of SOCS6 was significantly associated with tumor size $(P=0.001)$. Then according to Kaplan-Meier curve analysis, low expression of SOCS6 was significantly correlated with worse OS and RFS of GIST patients. Ultimately, it was revealed by Cox proportional regression model analysis that low expression of SOCS6 was an independent predictive factor for OS and RFS.

Conclusion: Low expression of SOCS6 was an independent prognostic factor for GIST, suggesting its potential as a novel biomarker predicting survival of GIST patients.

\section{Background}

As the most common stromal tumor originating from the gastrointestinal tract, gastrointestinal stromal tumor (GIST) accounts for $0.1-3 \%$ of all gastrointestinal malignant tumors and $6 \%$ of the sarcomas [1]. Globally, the annual incidence of GIST is 10/1000000 [2]. It has been revealed by genomic sequencing that activated mutations of receptor protein tyrosine kinase (RPTKs) or platelet-derived growth factor receptor-a(PDGFRA) occur in approximately $85-90 \%$ of GISTs [3]. Although the application of receptor kinase KIT and PDGFRA inhibitor, imatinib, could efficiently control the progression of $80-90 \%$ GISTs, about $50 \%$ of GIST patients experience secondary resistance against imatinib within 2 years [4-5]. As far as we are concerned, curative surgery remains the primary treatment for resectable GISTs despite the fact more than $50 \%$ of patients with advanced GISTs will experience tumor recurrences [2]. Thus, seeking more biomarkers associated with prognosis and clinicopathological features of GISTs is still a meaningful work for us.

Abnormally persistent activation of growth factor receptor signaling pathways has been reported to participate in a series of pathological processes such as autoimmune diseases and malignant tumors. Negative feedback regulation plays vital roles in maintaining the balance between pro-proliferative signals and anti-proliferative signals. Persistent pro-proliferative signals triggered by loss of function (LOF) of the negative feedback regulation mechanisms would lead to excessive proliferation of cells and even occurrence of malignant tumors. As one of the ubiquitous E3 ubiquitin ligases, suppressor of cytokine signaling 6 (SOCS6) could promote the ubiquitin-mediated degradation of proteins by binding 
with phosphorylated tyrosine receptors or signaling proteins [6, 7]. The locus (18q22.2) where human SOCS6 resides is commonly associated with malignant tumors [8]. Deletion of the genes located at 18q22.2 have been reported to occur in multiple malignant tumors such as lung spuamous cell carcinoma, hepatocellular carcinoma, prostate cancer, leukemia and this deletion is significantly associated with poor prognosis [9-12]. However, it remains unclear whether SOCS6 affects survival of GIST patients. Thus, we performed the present study to assess the expression of SOCS6 in GIST tissues and explore the capability of SOCS6 expression to predict prognosis of GIST patients.

\section{Materials And Methods}

\section{Patients and clinical samples}

Patients having received curative surgery and been diagnosed with GIST at the Department of Gastrointestinal Surgery, The First Affiliated Hospital, Sun Yat-sen University between January 2000 and December 2014 were retrospectively screened. The diagnosis of GIST was made when histopathological features of GIST was met and CD117 and/or CD34 positivity was confirmed by immunohistochemical staining. The inclusion criteria of this study were as follows: curative resection; no distant metastasis; no preoperative TKI application; without other malignant tumors; complete clinicopathological data. The following information of each included patients were retrieved from the medical records: gender, age, tumor size, necrosis of tumor, mitotic index (per 50 high-power fields). Tumor risk grade of each patient was assessed according to the modified National Institutes of Health $(\mathrm{NIH})$ consensus [13]. Declaration of Helsinki was adhered to during the whole process of this study. Informed consent in written form had obtained from each individual patient before the study.

\section{Immunohistochemical staining and scoring}

The expression level of SOCS6 in GIST tissue was evaluated by immunohistochemical staining. Slides bearing GIST tissues were initially embedded in paraffin. The GIST tissues were first deparaffined by xylene and then rehydrated using alcohol of different concentrations $(100 \%, 95 \%, 85 \%$ and $75 \%)$. Then after rehydration, the slides were soaked in $0.3 \%$ hydrogen peroxide for 20 minutes to block endogenous peroxidase activity. And the GIST tissues were blocked by $10 \%$ bovine serum albumin (BSA) for 30 minutes. Subsequently, GIST tissues were incubated with SOCS6-specific antibody (1:100, ab197335, Abcam, Cambridge, MA, USA) at $4{ }^{\circ} \mathrm{C}$ overnight. On the second day, GIST tissues were incubated with the biotinylated secondary antibody for 30 minutes. Subsequently, the 3,3,-diaminobenzidine (DAB) (GK600510, Genomics Shanghai, China) was used as the chromogenic substrate to help visualize the antibody-conjugated SOCS6. Ultimately, the GIST tissues were counter stained using hematoxylin. The stained GIST tissues were semi-quantitatively scored by two independent pathologists without priorly knowing patients, clinicopathological information. Staining intensities were as follows: strong staining (3, shown in Fig. 1a), moderate staining (2, shown in Fig. 1b), weak staining (1, shown in Fig. 1c) and negative staining ( 0 , shown in Fig. $1 \mathrm{~d})$. While positive cell percentage scores were defined as follows: 0 (< $5 \%), 1(5-24 \%), 2(25-50 \%)$ and $3(>50 \%)$. Finally, the scores were obtained by multiplying the staining 
intensity score and positive cell percentage score. High expression was defined when the total score was $\geq 4$ and $<4$ was defined as low expression.

\section{Statistical analysis}

The STATA14.0 software (Stata Corp LP, College Station, Texas) was adopted to accomplished the aforementioned statistical analyses. Measurement data were compared by $T$ test while categorical variables using $\chi 2$ test. Overall survival (OS) was defined as the duration between curative surgery and death no matter the cause while the time between curative surgery and tumor recurrence was recorded as recurrence-free survival (RFS). The Kaplan-Meier curve analysis was performed to estimate survival of GIST patients, which was further tested by log-rank test. Both univariate and multivariate Cox proportional hazard regression model analyses were accomplished to calculate hazard ratio (HR) and $95 \%$ confidential interval $(95 \% \mathrm{Cl})$ and to identify independent prognostic factors for GIST patients. All the tests accomplished in this study were two-sided in nature and a $\mathrm{P}$ value $<0.05$ was considered as statistically significant.

\section{Results}

\section{The associations between SOCS6 expression and clinicopathological variables}

After accomplishing immunohistochemical staining, we could find that SOCS6 was mainly distributed in cytoplasm and nucleus. Of the 255 GIST patients, 102 ones were identified to have high SOCS6 and 153 ones low SOCS6 expression. The associations between SOCS6 expression and clinicopathological variables were presented in Table 1, from which we could see that SOCS6 expression was significantly associated with tumor size $(P=0.001)$. However, SOCS6 expression was not significantly correlated with other variables including age, gender, tumor location, necrosis of tumor, mitotic index and NIH risk grade. 
Table 1

Associations between SOCS6 expression and clinicopathological variables.

\begin{tabular}{|llll|}
\hline & $\begin{array}{l}\text { High expression } \\
(\mathbf{N}=102)\end{array}$ & $\begin{array}{l}\text { Low expression } \\
(\mathbf{N}=153)\end{array}$ & $\mathbf{P}$ \\
\hline Age & $54.45 \pm 12.70$ & $56.42 \pm 12.28$ & 0.22 \\
\hline Gender & & & 0.44 \\
\hline Male & $55(53.9 \%)$ & $91(59.5 \%)$ & \\
\hline Female & $47(46.1 \%)$ & $62(40.5 \%)$ & \\
\hline Tumor location & & & 0.48 \\
\hline Stomach & $66(64.7 \%)$ & $94(61.4 \%)$ & \\
\hline Small intestine & $31(33.3 \%)$ & $51(33.3 \%)$ & \\
\hline Colorectum & $2(2.0 \%)$ & $8(5.2 \%)$ & 0.001 \\
\hline Tumor size(cm) & $4.97 \pm 4.13$ & $7.86 \pm 6.05$ & 0.58 \\
\hline Necrosis of tumor & & & \\
\hline No & $73(71.6 \%)$ & $104(68.0 \%)$ & \\
\hline Yes & $29(28.4 \%)$ & $49(32.0 \%)$ & \\
\hline Mitotic index & & & \\
\hline$<5 \%$ & $65(63.7 \%)$ & $92(60.1 \%)$ & \\
\hline $5 \% \sim 10 \%$ & $20(19.6 \%)$ & $26(17.0 \%)$ & \\
\hline$>10 \%$ & $17(16.7 \%)$ & $35(22.9 \%)$ & \\
\hline NIH risk grade & & & \\
\hline Extremely low & $12(11.8 \%)$ & $12(7.8 \%)$ & \\
\hline Low & $28(27.5 \%)$ & $39(25.5 \%)$ & \\
\hline Moderate & $21(20.6 \%)$ & $35(22.9 \%)$ & \\
\hline High & $41(40.2 \%)$ & $67(43.8 \%)$ & \\
\hline & & & \\
\hline S & & & \\
\hline
\end{tabular}

\section{The impacts of SOCS6 on OS}

The follow-ups of the 255 GIST patients range from 2 to 156 months and the 1-year, 3-year and 5-year OS of these 255 patients were $96.81 \%, 92 \%$ and $83.1 \%$ respectively. The mean survival time of 255 patients was $119.82 \pm 4.38$ months. For patients with high SOCS6 expression, the 1-year, 3-year and 5-year OS were $97.79 \%, 95.75 \%$ and $89.62 \%$ respectively and the mean survival was $135.00 \pm 5.30$ months. 
Whereas, for patients with low SOCS6 expression, the 1-year, 3-year and 5-year OS were $96.04 \%, 89.49 \%$ and $78.95 \%$ respectively and the mean survival was $95.56 \pm 4.56$ months. According to Kaplan-Meier curve analysis, low SOCS6 expression $(P=0.0059)$, tumor size $>5 \mathrm{~cm}(P<0.001)$, necrosis of tumor $(P<$ $0.001)$, Mitotic index $>5 / 50 \mathrm{HPF}(P=0.0001)$, moderate or high NIH risk grade $(P<0.001)$ were significantly associated with worse OS of GIST patients (shown in Fig. 2a to Fig. 2e). Then it was revealed by univariate Cox proportional hazard regression model analysis that tumor size $(\mathrm{P}<0.001, \mathrm{HR}=$ 3.67, 95\%Cl: 1.99 6.74), necorsis of tumor $(\mathrm{P}<0.001, \mathrm{HR}=4.50,95 \% \mathrm{Cl}: 2.48 \sim 8.18)$, mitotic index $(\mathrm{P}<$ $0.001, \mathrm{HR}=3.13,95 \% \mathrm{Cl}: 1.75 \sim 5.62)$, moderate or high $\mathrm{NIH}$ risk grade $(\mathrm{P}<0.001, \mathrm{HR}=7.37,95 \% \mathrm{Cl}$ : 2.90 18.73) and low SOCS6 expression ( $P=0.008, H R=2.51,95 \% \mathrm{Cl}: 1.27 \sim 4.93)$ were significantly associated with OS of GIST patients (shown in Table 2). Subsequently, tumor size $(P=0.007, H R=2.49$, 95\%Cl: 1.27 4.56), necorsis of tumor ( $\mathrm{P}=0.001, \mathrm{HR}=3.03,95 \% \mathrm{Cl}$ : $1.54 \sim 5.95)$, mitotic index $(\mathrm{P}=0.023$, $\mathrm{HR}=2.11,95 \% \mathrm{Cl}: 1.11 \sim 4.02)$, and low SocS6 expression ( $\mathrm{P}=0.009, \mathrm{HR}=2.58,95 \% \mathrm{Cl}: 1.27 \sim 5.24)$ were demonstrated by multivariate Cox proportional hazard regression model analysis to be independent predictive factors for OS (shown in Table 2). 
Table 2

Cox proportional-hazard regression model analysis for overall survival.

Univariate

3-year $\quad$ 5-year $\quad H R(95 \% \mathrm{Cl})$

OS

Age(years)

$\leq 65$

$>65$

Gender

Male

Female

Tumor location

Stomach

Non-stomach

$91.14 \%$

$93.43 \%$

$86.00 \%$ reference

1.75(0.98 3.11)

0.058

reference

Tumor size(cm)

\begin{tabular}{lllllll}
$\leq 5$ & $96.17 \%$ & $90.07 \%$ & reference & \multicolumn{3}{l}{ reference } \\
$>5$ & $86.41 \%$ & $73.38 \%$ & $3.67(1.99 \sim 6.74)$ & $<$ & $2.40(1.27 \sim 4.56)$ & 0.007
\end{tabular}

\section{Multivariate}

$\mathrm{HR}(95 \% \mathrm{Cl})$

$\mathrm{P}$

reference

1.54(0.81 2.93)

0.187

reference

$0.61(0.32 \sim 1.12) \quad 0.115$

$1.20(0.66 \sim 2.20) \quad 0.550$
Necrosis of

tumor

\begin{tabular}{lllllll} 
No & $96.39 \%$ & $91.67 \%$ & reference & \multicolumn{3}{l}{ reference } \\
Yes & $82.47 \%$ & $66.62 \%$ & $4.50(2.48 \sim 8.18)$ & $<$ & $3.03(1.54 \sim 5.95)$ & 0.001
\end{tabular}

Mitotic index

\begin{tabular}{lllllll}
$\leq 5 \%$ & $96.47 \%$ & $89.78 \%$ & reference & \multicolumn{3}{l}{ reference } \\
$>5 \%$ & $84.74 \%$ & $72.18 \%$ & $3.13(1.75 \sim 5.62)$ & $<$ & $2.11(1.11 \sim 4.02)$ & 0.023
\end{tabular}

$\mathrm{NIH}$ risk grade

Extremely low or $\quad 100 \% \quad 100 \% \quad$ reference

low

Moderate or high

$87.41 \%$

$72.60 \%$

7.37(2.90 18.73)

$<$

0.001 socs6

expression 


\begin{tabular}{|lllllll|}
\hline & & & Univariate & & \multicolumn{2}{l|}{ Multivariate } \\
\hline High & $95.75 \%$ & $89.62 \%$ & reference & & reference & \\
\hline Low & $89.49 \%$ & $78.95 \%$ & $2.51(1.27 \sim 4.93)$ & 0.008 & $2.58(1.27 \sim 5.24)$ & 0.009 \\
\hline
\end{tabular}

\section{The impacts of SOCS6 expression on RFS}

The 1-year, 3-year and 5-year RFS of these 255 patients were $95.21 \%, 89.30 \%$ and $77.98 \%$ respectively. The median RFS of 255 patients was $114.02 \pm 4.61$ months. For patients with high SOCS6 expression, the 1-year, 3-year and 5-year RFS were $96.96 \%, 94.81 \%$ and $84.79 \%$ respectively and the mean RFS was $129.49 \pm 5.73$ months. Whereas, for patients with low SOCS6 expression, the 1-year, 3-year and 5-year RFS were $93.36 \%, 85.62 \%$ and $73.30 \%$ respectively and the mean RFS was $91.27 \pm 4.64$ months. According to Kaplan-Meier curve analysis, low SocS6 expression $(P=0.0071)$, non-stomach GIST $(P=$ 0.0107), tumor size $>5 \mathrm{~cm}(P<0.001)$, necrosis of tumor $(P<0.001)$, Mitotic index $>5 / 50 \mathrm{HPF}(P<0.001)$, moderate or high NIH risk grade $(P<0.001)$ were significantly associated with worse RFS of GIST patients (shown in Fig. 3a to Fig. 3f). Then it was revealed by univariate Cox proportional hazard regression model analysis that tumor size $(P<0.001, H R=3.18,95 \% \mathrm{Cl}: 1.84 \sim 5.50)$, tumor location $(P=0.012, H R=1.95$, 95\%Cl: 1.16 3.30), necorsis of tumor $(\mathrm{P}<0.001, \mathrm{HR}=3.93,95 \% \mathrm{Cl}: 2.31 \sim 6.68)$, mitotic index $(\mathrm{P}<0.001$, $\mathrm{HR}=2.93,95 \% \mathrm{Cl}: 1.73 \sim 4.97)$, moderate or high $\mathrm{NIH}$ risk grade $(\mathrm{P}<0.001, \mathrm{HR}=8.89,95 \% \mathrm{Cl}: 3.53 \sim 22.36)$ and low SOCS6 expression $(P=0.009, H R=2.20,95 \% \mathrm{Cl}: 1.22 \sim 3.98)$ were significantly associated with RFS of GIST patients (shown in Table 3). Subsequently, tumor size $(P=0.014, \mathrm{HR}=2.06,95 \% \mathrm{Cl}$ : 1.16 3.68), necorsis of tumor $(P=0.002, \mathrm{HR}=2.60,95 \% \mathrm{Cl}: 1.44 \sim 4.71)$, mitotic index $(P=0.020, \mathrm{HR}=$ 2.60, 95\% Cl: 1.44 4.71), and low SOCS6 expression ( $\mathrm{P}=0.018, \mathrm{HR}=2.10,95 \% \mathrm{Cl}: 1.14 \sim 3.89)$ were demonstrated by multivariate Cox proportional hazard regression model analysis to be independent predictive factors for RFS (shown in Table 3). 
Table 3

Cox proportional-hazard regression model analysis for for recurrence-free survival

Univariate

3-year 5-year $\quad \mathrm{HR}(95 \% \mathrm{Cl})$

RFS RFS

Age (years)

$\leq 65$

$>65$

Gender

Male

Female

Tumor location

Stomach

Non-stomach

$90.78 \%$

$86.79 \%$

$81.16 \%$

$72.53 \%$

1.95(1.16 3.30)

0.012

reference

Tumor size $(\mathrm{cm})$

$\leq 5$

$>5$

$94.86 \%$

$81.77 \%$

$65.76 \%$ reference

$3.18(1.84 \sim 5.50)$
Multivariate

$\begin{array}{lll}\text { P } & \mathrm{HR}(95 \% \mathrm{Cl}) & \mathrm{P}\end{array}$

$\begin{array}{lll}\text { P } & \mathrm{HR}(95 \% \mathrm{Cl}) & \mathrm{P}\end{array}$

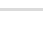




\begin{tabular}{|lllllll|}
\hline & & & Univariate & & \multicolumn{2}{l|}{ Multivariate } \\
\hline High & $94.81 \%$ & $84.79 \%$ & reference & & reference & \\
\hline Low & $85.62 \%$ & $73.30 \%$ & $2.20(1.22 \sim 3.98)$ & 0.009 & $2.10(1.14 \sim 3.89)$ & 0.018 \\
\hline
\end{tabular}

\section{Discussion}

As a member of cytokine signaling inhibitor protein family, SOCS6 is characterized by a functional Src homologous domain (SH2), a SOCS box located at the C-terminus, and a binding region of various length and sequence at the $\mathrm{N}$-terminus [8]. The $\mathrm{SH} 2$ domain is responsible for regulating cell signaling pathways via participating in the interaction between signaling proteins and phosphorylated tyrosine residues while the SOCS box serves as an elonginB/C-independent binding domain that links SOCS proteins to E3 ubiquitin ligases and proteasomes [8]. Currently, SOCS6 is considered as a specific regulator of receptor tyrosine kinase signaling pathway. Ba/F3, MEF and COS-7 cells, overexpression of SOCS6 could inhibit cell proliferation trough inhibiting expression of KIT and phosphorylation of ERK1/2 and p38 but does not affect the phosphorylation of AKT and STAT5 [15]. Thus, SOCS6 accomplishes its regulatory roles not only through ubiquitin-mediated degradation of receptor tyrosine kinases but also via negatively regulating downstream signaling proteins of receptor tyrosine kinase such as ERK1/2 and p38.

By far SOCS6 has been reported to be deleted in multiple malignant tumors. The absence of SOCS6 in primary lung squamous carcinoma had been reported to be significantly associated with worse survival of patients [9]. Yuan et al reported that expression of SOCS6 in prostate cancer was down-regulated and its low expression in prostate cancer was significantly associated with advanced stage and lymph node metastasis [11]. Furthermore, Yuan et al had also proven that low SOCS6 expression in prostate cancer was an independent prognostic factor [11]. Similarly, SOCS6 was down-regulated in hepatocellular carcinoma and the low expression of SOCS6 was significantly associated with progression, high recurrence risk and worse recurrence-free survival of hepatocellular carcinoma [10]. Besides its roles in lung squamous carcinoma, prostate cancer and hepatocellular carcinoma, SOCS6 could also inhibit the growth of gastric cancer, non-small cell lung cancer and cervical cancer via inhibiting angiogenesis, tumor cell proliferation and promoting apoptosis [16]. Furthermore, SOCS6 had been reported to regulate sensitivity of cancer cells to radiotherapy and chemotherapy [16-18]. And the epigenetic modification of the promoter region such as methylation has been proven to lead to down-regulation or loss of SOCS6 expression [19]. A more recent study has demonstrated that miR-k12-1-5p could lead to decreased expression of SOCS6 in Kaposi's sarcoma [20]. In glioblastoma, up-regulation of miR-494 could result in reduced expression of SOCS6 [21]. While in bladder cancer cells, IncRNA NBAT1 could regulate SOCS6 expression via miR-21-5p [22]. Thus, considering all these aforementioned studies, we could draw the conclusion that SOCS6 could act as a tumor suppressor gene in multiple cancers and the expression of SOCS6 was regulated by methylation of its promoter region and was directly or indirectly controlled by miRNA and IncRNA. 
In the present study, it was revealed that SOCS6 expression in GIST was significantly associated with tumor size and was an independent prognostic factor for GIST patients. According to a study published in 2018, of the nine genes screened by CRISPR-Cas9 technology that were likely to lead to resistance against imatinib, SOCS6 was one of the most promising targets [23]. In the future, we will explore the associations between SOCS6 expression and proliferation and drug-resistance of GIST by performing invivo and in-vitro assays. Also, the mechanisms through which SOCS6 regulates proliferation and resistance against imatinib of GIST cells will also be investigated. However, some shortcomings of the present study are not totally to be neglected. Firstly, this study is a retrospective one in nature, meaning that selection bias is not absolutely avoidable. Secondly, the number of included patients is relatively small, warranting larger-scaled studies. Thirdly, the specific mechanisms have not been studied, suggesting further studies are needed to elucidate these mechanisms. Despite these drawbacks, the present study could still provide some valuable suggestions for future clinical practice and research given the fact that this is the first study reporting the prognostic significance of SOCS6 in GIST.

\section{Conclusion}

Low SOCS6 expression is an independent predictive factor for worse survival of GIST patients, suggesting its potential as a novel prognostic biomarker for GIST patients.

\section{Abbreviations}

SOCS6: suppressor of cytokine signaling 6; GIST: gastrointestinal stromal tumor; OS: overall survival; RFS: recurrence-free survival; PDGFRA: platelet-derived growth factor receptor-a; RPTK: receptor protein tyrosine kinase; LOF: Ioss of function; NIH: National Institutes of Health; TKL: tyrosine kinase inhibitor; BSA: bovine serum albumin; DAB: 3,3,-diaminobenzidine; HR: hazard ratio; 95\%Cl: 95\% confidential interval; HPF: high-power field; SH2: Src homologous domain.

\section{Declarations}

\section{Ethics approval and consent to participate}

This study was approved by the Ethics Committee of The Seventh Affiliated Hospital, Sun Yat-sen University and each individual patient had given his or her written informed consent.

\section{Consent for publication}

Not applicable. 


\section{Availability of data and materials}

The data analyzed in the present study are available from the corresponding author on reasonable requests.

\section{Competing interests}

The authors have no competing interests to declare.

\section{Funding}

The present study received support from the Sanming Project of Medicine in Shenzhen (SZSM201612022 to Z. L. and Y. W.)

\section{Authors' contributions}

$\mathrm{CHZ}, \mathrm{XHZ}$ and JOY designed the study. JOY, TLA and YW collected the data. JOY, TLA, YWZand XKW performed the IHC staining. JOY, TLA and XFL scored the stained slides. JOY, TLA, YW and YWZ accomplished the statistical analyses. JOY, TLA and YW drafted and revised the manuscript. All authors read and approved of the final manuscript.

\section{Acknowledgements}

The authors would thank Lyujia Cheng and Cheng Yuan for their great help during this study.

\section{References}

1. von Mehren M, Joensuu H. Gastrointestinal Stromal Tumors. J Clin Oncol. 2018;36(2):136-43.

2. Miettinen M, Lasota J. Gastrointestinal stromal tumors. Gastroenterol Clin North Am. 2013;42(2):399415.

3. Li GZ, Raut CP. Targeted therapy and personalized medicine in gastrointestinal stromal tumors: drug resistance, mechanisms, and treatment strategies. Onco Targets Ther. 2019;12:5123-33.

4. D K, JR Z. Current and emerging strategies for the management of imatinib-refractory advanced gastrointestinal stromal tumors. Ther Adv Med Oncol. 2012;4(5):255-70.

5. F L, H H, X L, DA R, Y W, R O, et al. FGFR-Mediated Reactivation of MAPK Signaling Attenuates Antitumor Effects of Imatinib in Gastrointestinal Stromal Tumors. Cancer Discov. 2015;5(4):438-51.

6. JU K, J S, B P, F Z, A F-M, L R. Suppressor of cytokine signaling 6 (SOCS6) negatively regulates Flt3 signal transduction through direct binding to phosphorylated tyrosines 591 and 919 of Flt3. J Biol 
Chem. 2012;287(43):36509-17.

7. Xia Y, Wei K, Yang FM, Hu LQ, Pan CF, Pan XL, et al. miR-1260b, mediated by YY1, activates KIT signaling by targeting SOCS6 to regulate cell proliferation and apoptosis in NSCLC. Cell Death Dis. 2019 Feb 8;10(2):112.

8. Kabir NN, Sun J, Rönnstrand L, Kazi JU. SOCS6 is a selective suppressor of receptor tyrosine kinase signaling. Tumour Biol. 2014;35(11):10581-89.

9. Sriram KB, Larsen JE, Savarimuthu Francis SM, Wright CM, Clarke BE, Duhig EE, et al. Arraycomparative genomic hybridization reveals loss of SOCS6 is associated with poor prognosis in primary lung squamous cell carcinoma. PLoS One. 2012;7(2):e30398.

10. Qiu X, Zheng J, Guo X, Gao X, Liu H, Tu Y, et al. Reduced expression of SOCS2 and SOCS6 in hepatocellular carcinoma correlates with aggressive tumor progression and poor prognosis. Mol Cell Biochem. 2013;378(1-2).

11. Liu J, Zheng Y, Gao J, Zhu G, Gao K, Zhang W, et al. Expression of SHP-1 and SOCS6 in patients with acute leukemia and their clinical implication. Onco Targets Ther. 2017;10:1915-20.

12. Yuan D, Wang W, Su J, Zhang Y, Luan B, Rao H, et al. SocS6 Functions as a Tumor Suppressor by Inducing Apoptosis and Inhibiting Angiogenesis in Human Prostate Cancer. Curr Cancer Drug Targets. 2018;18(9):894-904.

13. Joensuu $H$. Risk stratification of patients diagnosed with gastrointestinal stromal tumor. Hum Pathol. 2008;39(10):1411-19.

14. Tang $D$, Lin $T$, Wang $Y$, Cao H. High expression of proenkephalin is associated with favorable outcomes in patients with gastrointestinal stromal tumors. Cancer Manag Res. 2019;11:6681-90.

15. Bayle J, Letard S, Frank R, Dubreuil P, De Sepulveda P. Suppressor of cytokine signaling 6 associates with KIT and regulates KIT receptor signaling. J Biol Chem. 2004;279(13):12249-59.

16. Sun X, Sun Y, Li J, Zhao X, Shi X, Gong T, et al. SOCS6 promotes radiosensitivity and decreases cancer cell stemness in esophageal squamous cell carcinoma by regulating c-Kit ubiquitylation. Cancer Cell Int. 2021;21(1):165.

17. Shen R, Wang Y, Wang C-X, Yin M, Liu H-L, Chen J-P, et al. MiRNA-155 mediates TAM resistance by modulating SOCS6-STAT3 signalling pathway in breast cancer. Am J Transl Res. 2015;7(10):211526.

18. Wang XJ, Zhang DL, Fu C, Wei BZ, Li GJ. MiR-183 modulates multi-drug resistance in hepatocellular cancer (HCC) cells via miR-183-IDH2/SOCS6-HIF-1a feedback loop. Eur Rev Med Pharmacol Sci. 2016;20(10):2020-27.

19. Lai R-H, Hsiao Y-W, Wang M-J, Lin H-Y, Wu C-W, Chi C-W, et al. SOCS6, down-regulated in gastric cancer, inhibits cell proliferation and colony formation. Cancer Lett. 2010;288(1):75-85.

20. Zhang J, Pu X-M, Xiong Y. kshv-mir-k12-1-5p promotes cell growth and metastasis by targeting SOCS6 in Kaposi's sarcoma cells. Cancer Manag Res. 2019;11:4985-95. 
21. Ou-Yang Q, He X, Yang A, Li B, Xu M. Interference with NTSR1 Expression Exerts an Anti-Invasion Effect via the Jun/miR-494/SOCS6 Axis of Glioblastoma Cells. Cell Physiol Biochem. 2018;49(6):2382-95.

22. Liu Z, Xie D, Zhang H. Long noncoding RNA neuroblastoma-associated transcript 1 gene inhibits malignant cellular phenotypes of bladder cancer through miR-21/SOCS6 axis. Cell Death Dis. 2018;9(10):1042.

23. Cao J, Wei J, Yang P, Zhang T, Chen Z, He F, et al. Genome-scale CRISPR-Cas9 knockout screening in gastrointestinal stromal tumor with Imatinib resistance. Mol Cancer. 2018;17(1):121-21.

\section{Figures}




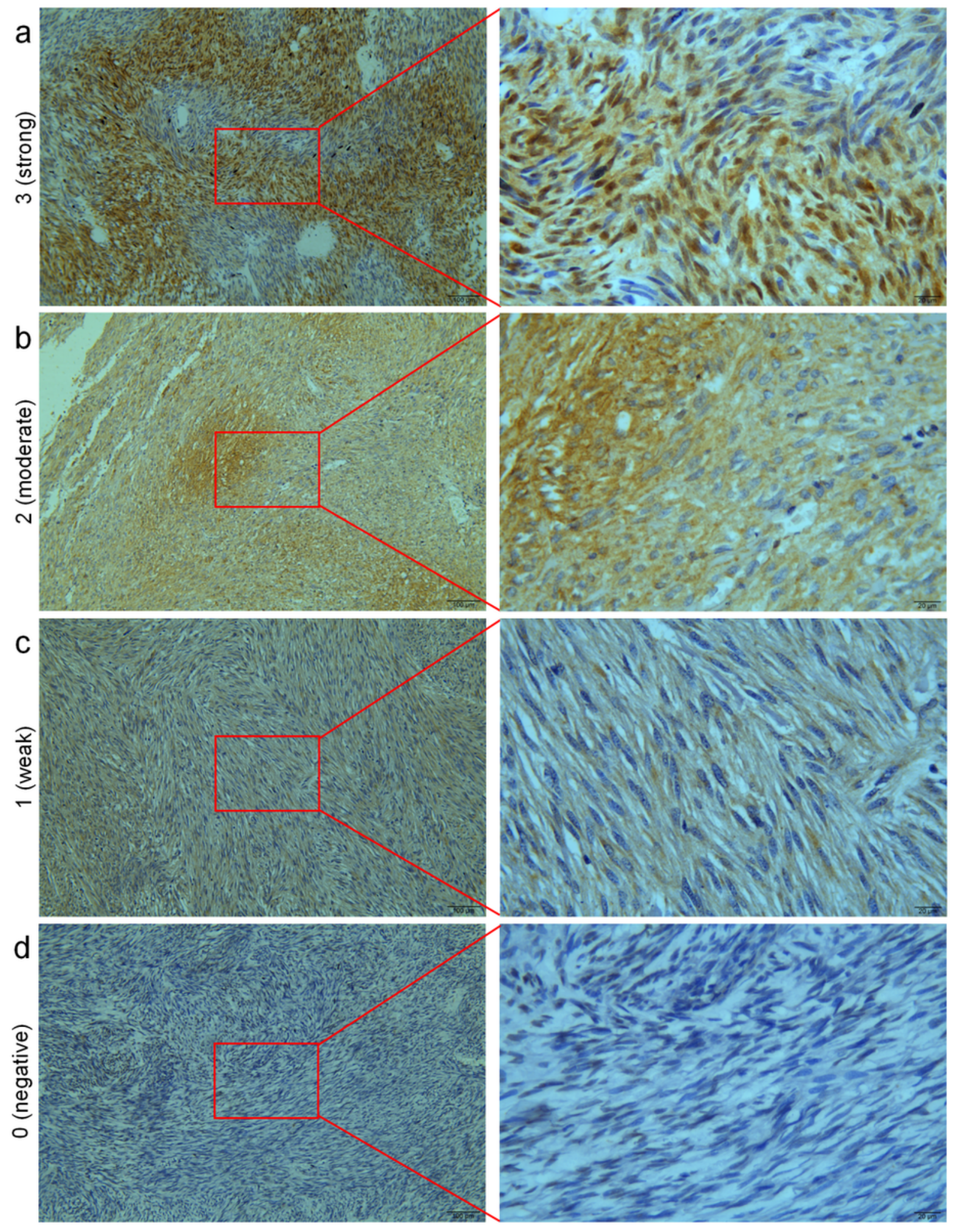

\section{Figure 1}

Different staining intensities of SOCS6 in GIST tissues identified by immunohistochemical staining. a: Strong staining intensity. b $\varangle$ Moderate staining intensity. c: Weak staining intensity. d: Negative staining intensity. 

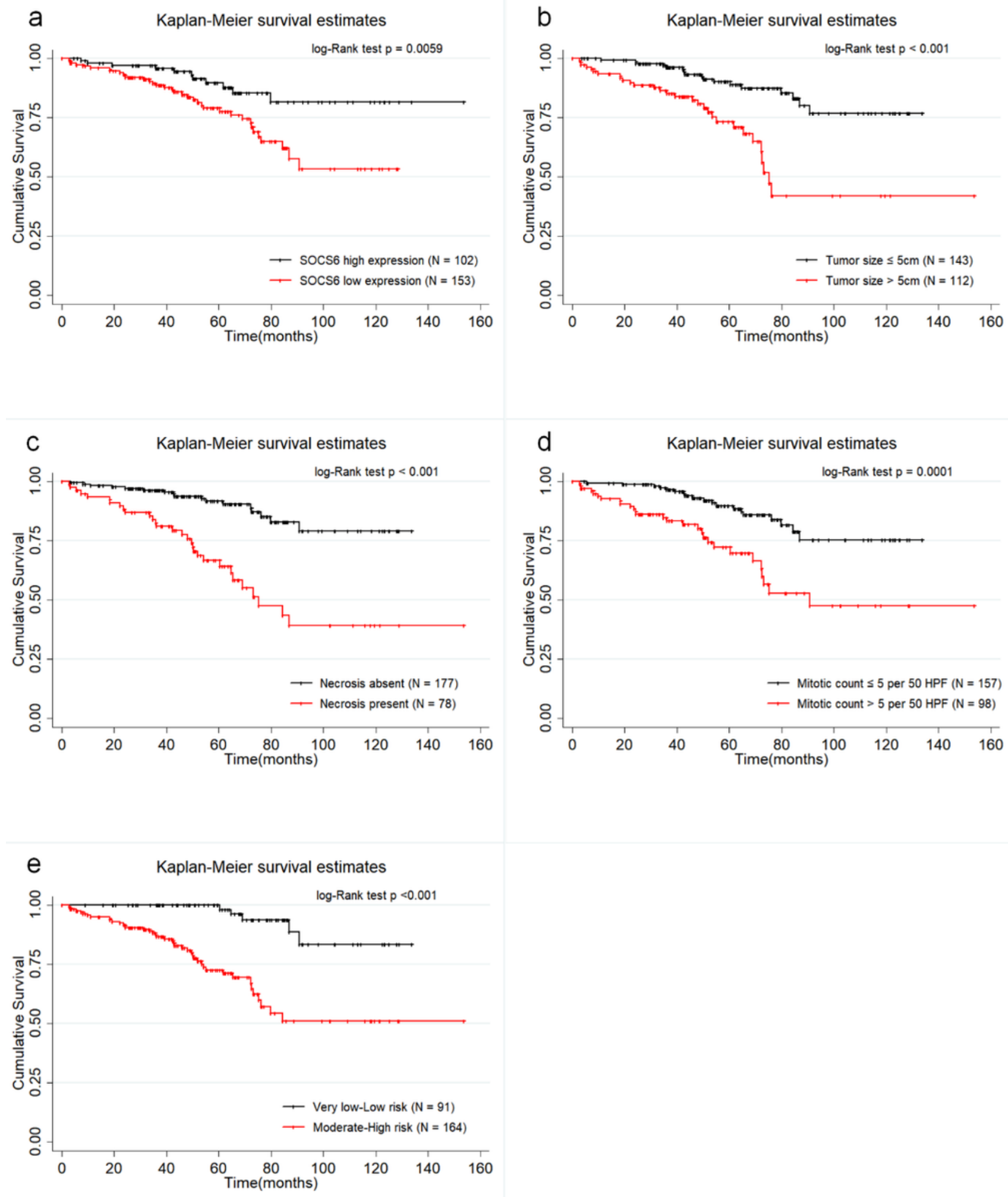

\section{Figure 2}

The impacts of SOCS expression, tumor size, necrosis of tumor, mitotic index, NIH risk grade on OS assessed according to Kaplan-Meier curve analysis and log-rank test. a: Impact of SOCS6 expression on OS of GIST patients. b: The impact of tumor size on OS of GIST patients. c: The impact of necrosis of tumor on OS of GIST patients. d: The impact of mitotic index on OS of GIST patients. e: The impact of $\mathrm{NIH}$ risk grade on OS of GIST patients. 

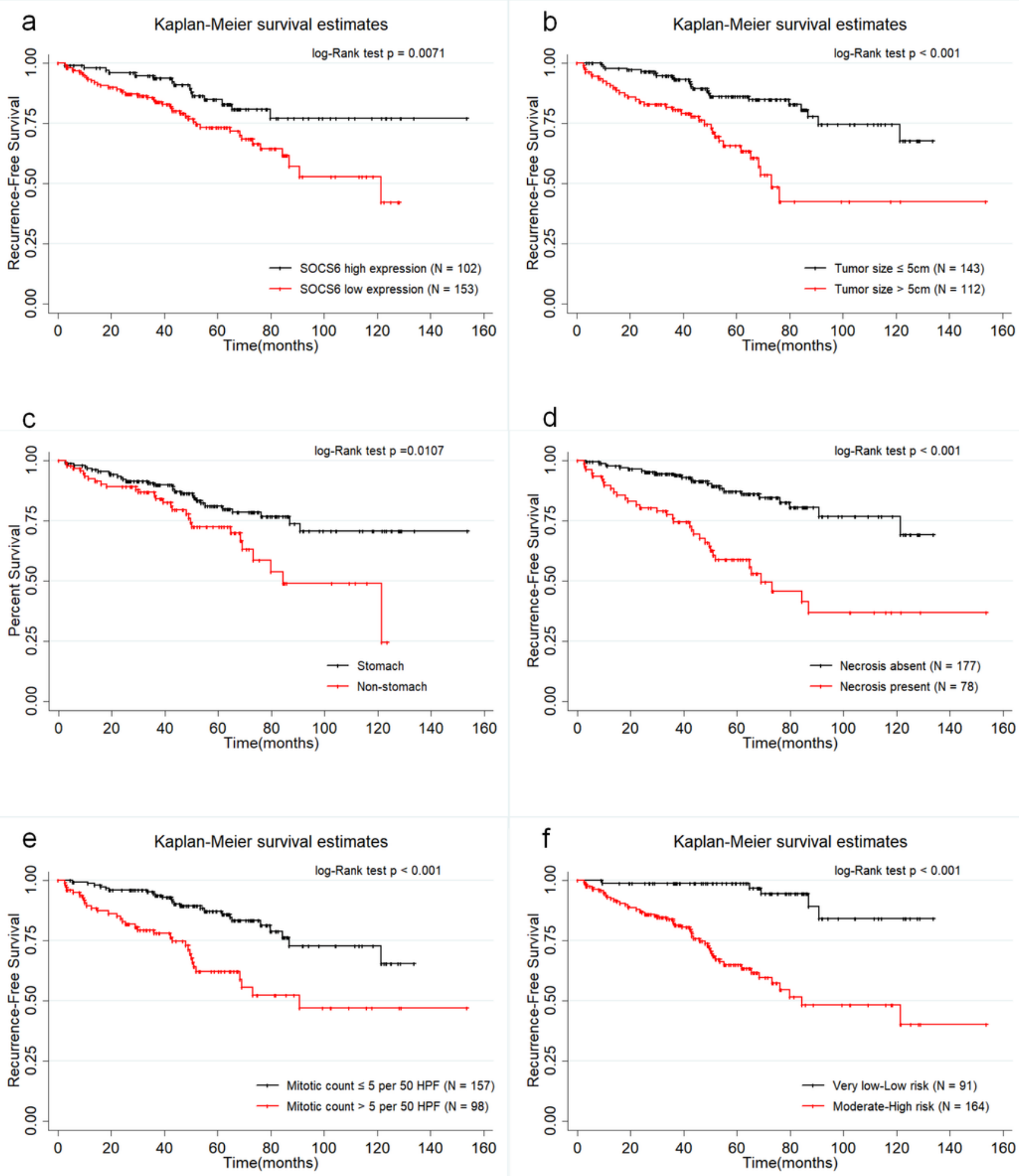

\section{Figure 3}

The impacts of SOCS expression, tumor size, necrosis of tumor, mitotic index, NIH risk grade on RFS assessed according to Kaplan-Meier curve analysis and log-rank test. a: Impact of SOCS6 expression on RFS of GIST patients. b: The impact of tumor size on RFS of GIST patients. c: The impact of tumor location on RFS of GIST patients. $d \otimes T$ The impact of necrosis of tumor on RFS of GIST patients. e: The impact of mitotic index on RFS of GIST patients. f: The impact of NIH risk grade on RFS of GIST patients. 